\title{
On the study of the dynamic response in 3D additively manufactured auxetic structures
}

\author{
Niklas Fjeldberg ${ }^{2}$, Jesús Pernas ${ }^{1}$, David Varas $^{1}$, and Jordi Martín ${ }^{1}$ \\ ${ }^{1}$ Department of Continuum Mechanics and Structural Analysis, University Carlos III of Madrid, \\ 28911 Leganés, Madrid, Spain \\ ${ }^{2}$ Department of Mechanical Engineering, Østfold University College, 1757 Halden, Norway
}

\begin{abstract}
The main aim of this work is to analyse the quasi-static and the dynamic response and deformation behaviour of polymeric auxetic structures manufactured with an SLA (stereolitography) additive manufacturing technique. To this end, different experimental tests were performed using a universal servo-hydraulic instron machine and a Split Hopkinson Pressure Bar (SHPB). The study focuses on the understanding of the mechanical deformation response of the metamaterial depending on the type of load, the amount and distribution of the unit cells and the strain rate applied.
\end{abstract}

Keywords: Additive Manufacturing, Split Hopkinson Pressure Bar, Auxetic behaviour, Lattice structures, dynamic regime

\section{Introduction}

The pursuit of new materials and structures able to ensure a better mechanical performance at cheaper cost has been one of the main engineering concerns during last decades. These structures are meant to be evolving towards stronger, lighter and tougher components to face specific load conditions and boundary requirements. Thanks to the apparition of additive manufacturing a new scenario of possibilities has shown up. By means of this manufacturing technique, new geometrical configurations have been studied and launched the rise in the study of metamaterials and particularly auxetic based structures. An auxetic material is in simple terms a material with a negative Poisson's ratio. This fact provides them with an excellent shear strength [1,2] indentation resistance [1], high fracture toughness $[3,4]$ and a high energy dissipation capacity [5,6] which makes them especially suitable to certain applications such as shock absorbers, filters, composites, biomedical implants and can offer an enhanced protection for a large number of elements, structures and devices.

The study of the behaviour of this type of meta materials in dynamic regime is scarce, and this is the main objective of this study, unveil the driven parameters of behaviour under dynamic loading of the proposed auxetic lattice structure. 


\section{Unit cell geometry and parameters}

In the present work a re-entrant cell was considered for the study of auxetic behaviour. In figure 1 a picture of the representative unit cell in study, as well as the geometrical parameters necessary to define the unit cell are shown. It can be observed that the structure is composed by different cylinders connected through arms with a re-entrant angle, which causes the auxetic behaviour. This cell is selected due to its high stability in the axial direction of the cylinders, which will be the loading direction in the present study. The repetition of this cell produces the auxetic lattice and the samples to be tested thorough the experimental campaign.
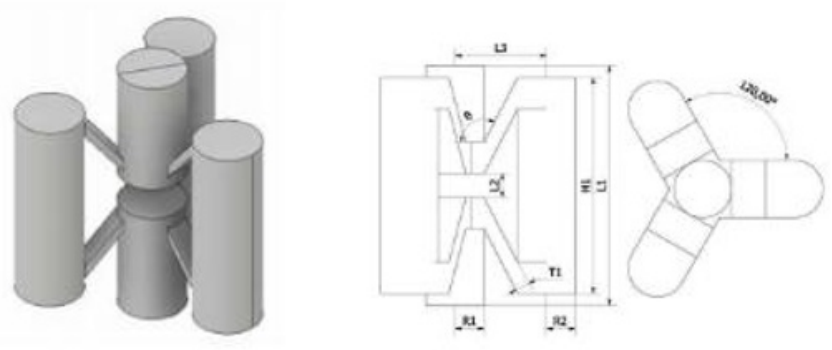

Figure 1: Unit cell purposed for the study and its parameters.

\section{Experimental setups}

For the development of all the experiments, several auxetic pseudo-material lattices will be produced by means of a 3D stereolithography printer using the commercial Durable polymeric resin by Formlabs. It has been proved in previous studies [7] that sample manufacturing through this technique leads to the obtention of high quality and high repeatability parts with final properties that doesn't depend on the printing parameters.

Two different types of tests were be carried out on the lattices: quasistatic compression and Split hopkinson pressure bar test for the dynamic response.

\subsection{Quasistatic compression}

The compression tests were carried out in a servo-hydraulic equipment with a $10 \mathrm{kN}$ load cell. The specimens were tested at different strain rates using two different cross head speeds, 0.02 and $0.2 \mathrm{~mm} / \mathrm{s}$.

All tests were recorded with a camera, so the displacements were obtained from three different and independent sources: a tracking of the area of the lattice with a computer-based software, with the displacement of the machine itself and with an external LVDT (Linear Variable Differential Transformer) device. These displacements were compared to ensure a triple check of the measurements on control of the deformation. The force is measured using a load cell.

For the quasistatic test, the lattice used is shown in Figure 2 from an isometric point of view as an example. 


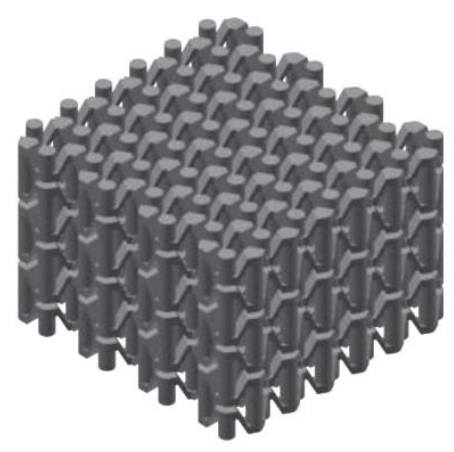

Figure 2: Isometric view of the lattice used in the quasi-static compression test.

\subsection{Split Hopkinson Bar/Kolsky bar}

The Split Hopkinson Pressure Bar (SHPB) was used to test the lattices at a high strain rate by imposing a dynamic load. The diameter of the bars limits the samples to be tested, the experimental apparatus in the Uc3m Lab consists in $22 \mathrm{~mm}$ diameter bars, so the specimens could not be greater than this diameter. So the lattice size for this experimental setup was adapted for the setup, being the same dimensions in the $\mathrm{x}$ and $\mathrm{y}$-axis. In the $\mathrm{z}$-axis the lattices specimens had different number of layers of unit cells, increasing its length and thus decreasing the compression deformation rate. In figure 3 a four-layer lattice is shown as an example of the final appearance and shape of the samples that has been tested. The velocity of the SHPB striker was selected varying the gas gun pressure and the induced strain by the impact was measured by strain gauges at the bar at $1 \mathrm{MHz}$. The complete deformation of the specimens was recorded using an ultra-high-speed camera (Photron SA-Z), whilst the illumination was provided by in-house design high power LED spotlights. This setup also allows to measure the movement of the bars using a computer vision tracking software to compare the displacement with the SHPB theory.

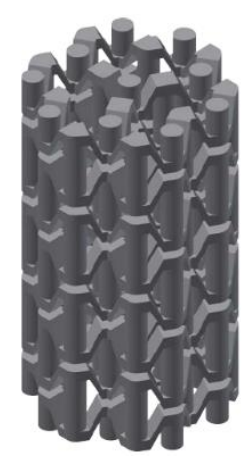

Figure 3: Isometric view of a four-layered lattice for used in SHPB test. 


\section{Results and Conclusions}

\subsection{Quasistatic compression}

Figure 4 shows a pair of the representative force-displacement and displacement-time type of curves that have been obtained of the auxetic structure under quasistatic compression. It can be observed how the displacement measured using the different techniques shows very good agreement, thus the setup is stiff enough to compress the sample without suffer an appreciable displacement. From the force displacement curve, it can be observed how the force increase with the imposed displacement up to a point in which the lattice suffers an instability a loose its bear capacity.

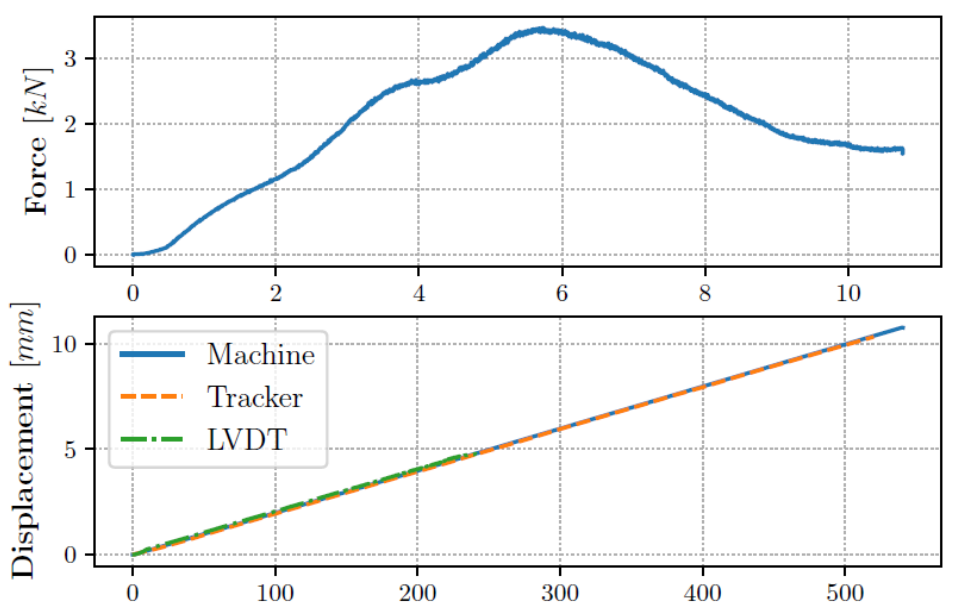

Figure 4: Force-displacement and displacement-time example curves obtained in the quasistatic compression tests.

\subsection{Split hopkinson Pressure Bar}

In figure 5 some images obtained in the SHPB experimental setup can be seen as an example. In this figure three frames at different times during the test are shown: the first frame of the recording, half time of the total frames and the last one. It can be observed how the specimens compress under the dynamic loading, without any buckling effect, the last frame shows the recovery of the specimen up to almost the original length, showing its outstanding recovery behaviour under dynamic loading. 


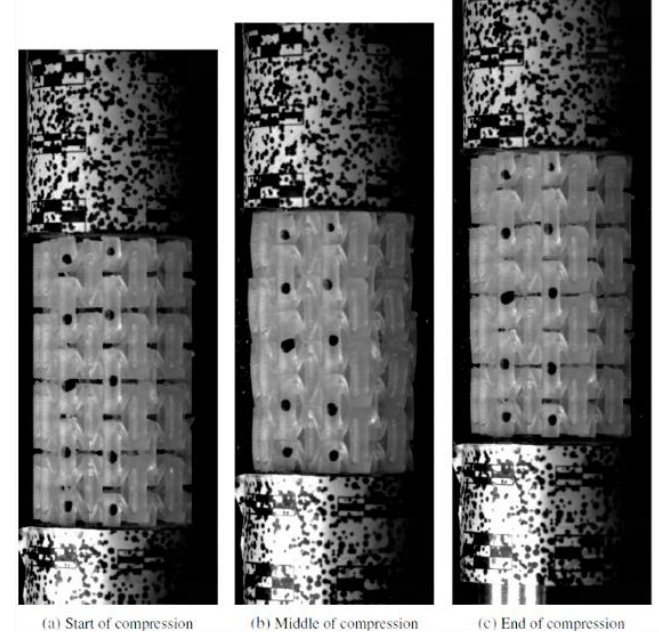

Figure 5: Examples of images obtained in SHPB test.

\subsection{Results}

The performed tests showed that geometrical parameters of the unit cell have influence on the behaviour of the whole lattice, having some of them more effect than others.

In addition, both the strain rate and the number and disposition of the unit cells in the lattice had an influence on the deformation mechanisms. All the aforementioned factors can control the total amount of energy that the structure is capable to absorb when external loads are applied.

\section{Acknowledgments}

This research was funded by Ministerio de Asuntos Económicos y Transformación Digital, Gobierno de España grant number DPI2017-85073-R, and Vicerrectorado de Política Científica UC3M (Projects 2013/00413/003 and 2014/00006/003). 


\section{References}

1. R. Lakes, foam structures with a negative Poisson's ratio, Science 27235 (4792) (1987) 1038-1040.

2. F. Scarpa, P. J. Tomlin, On the transverse shear modulus of negative Poisson's ratio honeycomb structures, Fatigue Fract Eng M , 23 (8) (2000) 717-720

3. R.S. Lakes, 1993. Design considerations for materials with negative Poisson's ratios. J. Mech. Des. 115 (1993) 696-700.

4. M. Bianchi, F.L. Scarpa, C.W. Smith, Stiffness and energy dissipation in polyurethane auxetic foams, J. Mater. Sci. 43 (2008) 5851-5860.

5. A. Bezazi, F. Scarpa, Mechanical behaviour of conventional and negative Poisson's ratio thermoplastic polyurethane foams under compressive cyclic loading, Int. J. Fatigue 29 (2007) 922-930.

6. F. Scarpa, P. Pastorino, A. Garelli, S. Patsias, M. Ruzzene, Auxetic compliant flexible PU foams: static and dynamic properties, Phys. Status. Solidi (B) 242 (2005) 681-694.

7. J. Martínn-Montal, J. Pernas-Sánchez, D. Varas, Experimental characterization framework for SLA additive manufacturing materials, Polymers 13 (7), tex.articlenumber: $\quad 1147 \quad$ (2021). doi:10.3390/polym13071147. URL https://www.mdpi.com/2073-4360/13/7/1147 